\title{
A NATURAL COMPANION TO CATALAN'S CONSTANT
}

\section{John M. Campbell, Paul Levrie* and Amrik Singh Nimbran}

Abstract. We consider here a trilogarithmic expression that plays a similar role to Catalan's constant $G$ in many ways:

$$
\mathscr{G}:=\mathfrak{I}\left(\operatorname{Li}_{3}\left(\frac{i+1}{2}\right)\right) .
$$

The main purpose of this article is to demonstrate how $\mathscr{G}$ is a naturally occurring and useful expression that deserves to be recognized as a mathematical constant and as a natural trilogarithmic "extension" of Catalan's constant $G$. Having identified this constant, we evaluate many new and non-trivial integrals, Euler-type sums, ${ }_{p} F_{q}$ series, and binomial-harmonic series using $\mathscr{G}$, extending known results on the classical version of Catalan's constant.

Mathematics subject classification (2020): 33B30, 33C20, 33B15.

Keywords and phrases: Catalan's constant, trilogarithm, symbolic evaluation, Euler-type sum.

\section{REFERENCES}

[1] V. S. ADAMCHIK, 33 representations for Catalan's constant, https://web.archive.org/web/20160807111945/https://www.cs.cmu.edu/ adamchik/ articles/catalan/catalan.htm.

[2] V. S. ADAMCHIK, A certain series associated with Catalan's constant, Z. Anal. Anwendungen, 21 (2002) 817-826.

[3] V. S. AdAMchiK, On Stirling numbers and Euler sums, J. Comput. Appl. Math., 79 (1997) 119-130.

[4] C. C. AdAMs, The newest inductee in the number hall of fame, Math. Mag., 71 (1998) 341-349.

[5] B. C. Berndt, Ramanujan's notebooks. Part I, Springer-Verlag, New York (1985).

[6] J. M. CAMPBELL, New series involving harmonic numbers and squared central binomial coefficients, Rocky Mountain J. Math., 49 (2019) 2513-2544.

[7] J. M. CAMPBELL, Series containing squared central binomial coefficients and alternating harmonic numbers, Mediterr. J. Math., 16 (2019) Art. 37, 7.

[8] J. M. CAMpbell, M. CANTARIni AND J. D'Aurizio, Symbolic computations via Fourier-Legendre expansions and fractional operators, Integral Transforms Spec. Funct., (2021).

[9] J. M. CAMPBell, J. D'Aurizio And J. Sondow, On the interplay among hypergeometric functions, complete elliptic integrals, and Fourier-Legendre expansions, J. Math. Anal. Appl., 479 (2019) 90-121.

[10] M. CANTARIni And J. D'Aurizio, On the interplay between hypergeometric series, FourierLegendre expansions and Euler sums, Boll. Unione Mat. Ital., 12 (2019) 623-656.

[11] M. W. Coffey, Alternative evaluation of a ln tan integral arising in quantum field theory, Math. Phys. Anal. Geom., 13 (2010) 191-204.

[12] M. W. COFFEY, Evaluation of a ln tan integral arising in quantum field theory, J. Math. Phys., 49 (2008), 093508, 15.

[13] L. Euler, Meditationes circa singulare serierum genus, Novi Commentarii academiae scientiarum Petropolitanae, 20 (1776) 140-186.

[14] F. FerRetti, A. Gambini And D. Ritelli, Identities for Catalan's Constant Arising from Integrals Depending on a Parameter, Acta Math. Sin. (Engl. Ser.), 36 (2020) 1083-1093.

[15] S. R. FInCH, Central Binomial Coefficients (2007), http://compmath.files.wordpress.com/2008/08/central_binomial_coefficients_ finch.pdf. 
[16] S. R. FINCH, Correlation between Angle and Side (2017), arXiv:1012.0781.

[17] B. GourÉvitch, The world of $\pi$, http://www . pi314.net/eng/hypergse13.php.

[18] I. S. Gradshteyn And I. M. RyzhiK, Table of Integrals, Series, and Products, 7e, Academic Press/Elsevier (2007).

[19] G. Huvent, Formules BBP (2001), http://www. lacim.uqam.ca/ plouffe/articles/huvent_seminaire.pdf.

[20] L. Lewin, Polylogarithms and Associated Functions, Elsevier North Holland, New York (1981).

[21] R. E. MiLES, Random points, sets and tessellations on the surface of a sphere, Sankhyā Ser. A, 33 (1971) 145-174.

[22] A. S. Nimbran, Deriving Forsyth-Glaisher type series for $\frac{1}{\pi}$ and Catalan's constant by an elementary method, Math. Student, 84 (2015) 69-86.

[23] N. J. A. SLOANE, The On-line Encyclopedia of Integer Sequences, http://oeis org.

[24] A. Sofo AND A. S. Nimbran, Euler-like sums via powers of log, arctan and arctanh functions, Integral Transforms Spec. Funct., 31 (2020), 966-981.

[25] J. SPIESS, Some identities involving harmonic numbers, Math. Comp., 55 (1990) 839-863.

[26] X. WANG AND W. CHU, Further Ramanujan-like series containing harmonic numbers and squared binomial coefficients, Ramanujan J., 52 (2020) 641-668. 